\title{
Assessment of Drugs’ Donation Practices in Sudan
}

\author{
Jacob L. Doro ${ }^{1}$, Asim F. Mustafa ${ }^{2}$, Ahmed S. Eldalo ${ }^{3}$, Mirghani A. Yousif ${ }^{3,4^{*}}$ \\ ${ }^{1}$ Office of the South Sudan, Sudan Central Medical Supplies, Malakal, Sudan; ${ }^{2}$ Faculty of Pharmacy, Omdurman Islamic University, \\ Omdurman, Sudan; ${ }^{3}$ College of Pharmacy, Taif University, Taif, Saudi Arabia; ${ }^{4}$ Faculty of Pharmacy, University of Gezira, Wad \\ Medani, Sudan. \\ Email: *Mirghani53@yahoo.com
}

Received October $19^{\text {th }}, 2011$; revised November $14^{\text {th }}, 2011$; accepted December $16^{\text {th }}, 2011$

\begin{abstract}
The quality requirements of drug donation are different from those of other donated items. Purpose: To describe and assess key aspects of pharmaceutical donations in Sudan, the kinds of benefits and drawbacks associated with drug donations, and the government controlling role in the donation process. Methods: Retrospective and prospective studies were conducted in the period from April 2007 to Mar 2008 in the Federal Ministry of Health (FMOH) and some working NGOs' data bases. Some quality aspects of donated drugs were explored. Six randomly selected nongovernmental organizations (NGOs) were examined for the relevance and some aspects of quality of donated drugs such as whether the donated drugs were listed in the National Essential Drug List, or labeled in International Nonproprietary Names, their dosage forms, the language on the package and if the donated drugs were sampled or returned ones. Results: The study showed that most of the donated drugs were relevant to NEDL normative standards. The median times from the date of arrival to the date of expiration were more than 400 days. The time prior to expiration range between $44.8 \%$ and $47.5 \%$ were found less than one year in arrival. The top ten therapies were accounted for about 40 shipped items. The study was revealed that Sudan did not develop its own guidelines for drug donations and NGOs are making no effort to develop written donation policies. Conclusion: Development of national guidelines for drug donations is essential to control the donated drugs in Sudan.
\end{abstract}

Keywords: Assessment; Drug Donation; Practices; Sudan

\section{Introduction}

Drug donations are usually given in response to acute emergencies, but they can also be part of development aid. Donations may be given directly by governments, by non-governmental organizations (NGOs), as corporate donations (direct or through private voluntary organizations), or as private donations to single health facility. Although there are legitimate differences between these donations, basic rules should apply to them all [1].

The contribution of medicines in the form of donations to developing countries or disaster situations is often reviewed by communities in developed countries, as a useful way to provide much needed pharmaceutical supplies. As the lack of medicines is often presented as a pressing problem, an immediate donation of drugs is often perceived as the most pragmatic and direct response [2]. Many international organizations usually respond with drug donations to help meet the emergencies. Although the act of donating drugs may seem philanthropic and often genuinely, but the effects of inappropriate do-

"Corresponding author. nations or dumping can be downright dangerous on the receiving end. The quality requirements of drug products are different from those for other donated items, such as food and clothing. Drugs can be harmful if misused; they need to be identified easily through labels and written information using specially trained human resources, may need special storage conditions, and they should be destroyed in a professional way. Many donated drugs arrived unsorted and labeled in a language which is not easily understood. Some donated drugs came under trade names which are not registered for use in the recipient country, and without an International Nonproprietary Name (INN) or generic on the label. [3] Unfortunately, there are also many examples of drug donations which cause problems instead of being helpful. Hoen (1993) [4] had reported that eleven women in Lithuania temporary lost their eyesight after using a drug that had been provided through drug donations. The drug closantel was anthelmintic that should be used only in veterinary medicines but mistakenly given for the treatment of endometritis. Some donated drugs had been received without product information or package inserts, and doctors used 
to identify products by matching the names on the boxes with the names on the leaflets of other products. Beverley (2001) [5] had reported that in 1994, despite the existence of published Christian Medical Commission (CMC) guidelines for drug donations since 1980s; every emergency in Zaire produces new example of inappropriate donations. In Eastern Zaire, one relief organization chartered an airplane to deliver a huge shipment of commercial soft drink used by athletes, in the false belief that it could be used to treat people with cholera. In fact, this product can be dangerous if given to infants. In addition, the product was not only bulky and difficult to store, but caused considerable waste and was not cost-effective when compared with standard oral rehydration salt (ORS) therapies used to treat diarrhea. In Sudan, in 1990 a large consignment of drugs was sent from France to war-devastated Southern Sudan; each box contained a collection of small packets of drugs, some partly used. All were labeled in French, a language not spoken in Sudan. Most were inappropriate, some could be dangerous. There were: contact lens solutions, appetite stimulants, antidepressants and expired antibiotics and out of 50 boxes, 12 contained some of used drugs [3].

The prime objective of this Sudan first research attempt was to describe and to assess key aspects of pharmaceutical donations, the kinds of benefits and drawbacks associated with drug donations, and the government controlling role in the donation process.

\section{Materials and Methods}

\subsection{Classification Study}

The researchers were developed and illustrated a system for classifying donated drugs based on Sudan's Essential Drug List, and some criteria from the WHO Guidelines for Drug Donations as "normative standards" and were applied the systems to donated drugs. Donated drug products were classified as listed on the National Essential Drug List (NEDL), if a drug with the same active ingredient and in the same dosage-form regardless of its strength. The drug is to be classified as "relevant" when enlisted to treat a local disease. Each donated drug was counted once, even if the same product was shifted multiple times. Donated drug products that labeled in International Nonproprietary Name (INN) were classified as “relevant”. Each donated drug was counted separately. Donated drugs that labeled in locally understood language were classified as "relevant". WHO Guidelines for Drug Donations' Provision No. 8, specified that donations should be supplied in tablet dosage form [2]. Donated drugs were classified as relevant when the proportion of the tablet dosage forms were greater than the syrups and injections dosage forms.

A donated drug sample was classified as "inappropri- ate", when to be found as a returned one (drugs that been issued to patients and returned to a pharmacy or elsewhere).

\section{Development of Relevant Indicator Form Sheets}

A relevant indicators' form sheet was proposed and developed for collecting data regarding relevance of donated drugs to the local disease patterns. Some criteria from WHO Guidelines for Drug Donation and NEDL were used to measure to how extend the donated drugs were relevant. Six selected nongovernmental organizations (NGOs) were proposed by the Ministry of Humanitarian Affairs, Sudan as organizations that handle large quantities of donated drugs on a regular basis in Khartoum State. These organizations asked to be anonymous. For classification study, 300 samples (50 samples from each NGO health facility) were examined for the relevance and for some aspects of quality. The collected data were analyzed by using SPSS, Version 14.

\subsection{Qualitative Study}

Quality indicators form sheet was developed for recording data regarding quality of donated drugs to show the date of arrival, expiry date and remaining shelf life of the donated item. Retrospective and prospective studies were conducted in the Federal Ministry of Health $(\mathrm{FMOH})$ and NGOs to explore the problems associated with drug donations' process in Khartoum State, within the period from Apr 2007 to Mar 2008. Inventory of receipt, shipment files and direct observation were made based on National Essential Drug List and some criteria from WHO Guidelines for Drug Donations as normative standards, a total of 2400 samples from two NGOs data base were collected. The two NGOs were chosen because they have good systems for recording data of the shipped items. Michael (1999) [6] Classification techniques were used to define the relevance of donated drugs.

\section{Methods of Analysis}

For classification study, the resultant values were modeled using Chi-square models to test the significances between different categories.

For quantitative study, the NGOs data aggregated into two-month time intervals to observe shifting patterns of drug donations and, in particular secular trends in the time to expiration of donations. For each of the twomonth time intervals; the median times between time of arrival and time to expiration for the donated products were calculated. These values were modeled using timesseries simple linear models to estimate any changes in median times that might have occurred over calendar time.

Standard list of key drugs was developed, which can 
be defined as standard list of the top ten drugs recommended for the treatment of most common health problems. The list based on specific essential drug list therapeutic category and applied the standard list of key drugs to the sampled donated products. The lists of sampled donated drugs were considered as "normative standard" when conforming to drug products represented therapies that were listed as top ten drugs.

\subsection{Donation Policy Study}

Face to face interviews were conducted with the medical coordinators for the six NGOs responded to the request at their premises, with the request for copies of their written donation policies. A set of questions were asked regarding criteria and procedures for product selection, donor-recipient communication, and logistics.

\subsection{Field Survey Study}

Face to face interviews and direct observation were conducted at NGOs health facilities, two persons were interviewed at each health facility one administrator and one health provider. Also other interviews were conducted with the Federal Ministry of Health and Central Medical Supplies: Director of International Health Department, Director of General Directorate of Pharmacy Administration, Director of Pharmacy Administration Khartoum State, and Deputy Director of Central Medical Supplies with the intention of including government officials involved in the drug donation process.

\section{Results}

The study showed that the mean proportion of donated drugs in NEDL was (91.8\%), and the proportion of donated drugs labeled in generic name or INN was (91.5\%). Most of the donated drugs in the current study were labeled in locally understood language (95.8\%). The donated drugs in tablet dosage form were $(90.2 \%)$, while that in syrup form were (5.6\%) and the injectable dosage form were (4.2\%). Out of the total donated drugs (99\%) were not drug samples and not returned or used ones.

Regarding the expiration of donated drugs; the time prior to expiration was examined at two NGOs data bases. Table 1 present's estimates of percentages of donated drugs with differing expiration times on arrival. The two NGOs were received somewhat shorter-dated drugs. Between 44.8\% (NGO A) and 47.5\% (NGO B) are said to have dating of less than one year on arrival which was not conforming to WHO Guidelines on Drug Donation.

Table 2 shows the results of more specific essential drug list therapeutic classification of NGO's donations. The top ten drug products accounting a significant proportion from $40.5 \%$ (NGO B) to $48.7 \%$ (NGO A) of do-
Table 1. Frequency distribution of donated drugs by dating status (NGOs).

\begin{tabular}{ccccc}
\hline \multirow{2}{*}{$\begin{array}{c}\text { Dating on arrival } \\
\text { at NGOs }\end{array}$} & \multicolumn{2}{c}{ NGO A } & \multicolumn{2}{c}{ NGO B } \\
\cline { 2 - 5 } & Number & \% & Number & \% \\
\hline < 3 months & 118 & 9.8 & 147 & 12.3 \\
3 - 6 months & 161 & 13.4 & 189 & 15.8 \\
$\mathbf{6}$-9 months & 115 & 9.6 & 88 & 7.3 \\
9 -11 months & 144 & 12.0 & 145 & 12.1 \\
$>$ 12 months & 662 & 55.2 & 630 & 52.5 \\
Total & 1200 & 100 & 1200 & 100 \\
\hline
\end{tabular}

Table 2. Percentage of shipment items for NGOs by specific NEDL category.

\begin{tabular}{cccc}
\hline Rank & Item & $\begin{array}{c}\text { Percent NGO } \\
\text { A }\end{array}$ & $\begin{array}{c}\text { Percent NGO } \\
\text { B }\end{array}$ \\
\hline 1 & Paracetamol & 8.4 & 10.4 \\
2 & Amoxicillin & 7.6 & 8.6 \\
3 & Artusenate & 6.4 & 5.3 \\
4 & Metronidazole & 6.0 & 4.6 \\
5 & Ferrous + folic & 5.8 & 3.5 \\
6 & Benzylpenicillin & 4.1 & 3.2 \\
7 & ORS & 3.9 & 2.7 \\
8 & Salbutamol & 2.4 & 2.2 \\
9 & Mebendazole & 2.1 & 0.0 \\
10 & Tetracycline eye ointment & 2.0 & 0.0 \\
Total & & 48.7 & 40.5 \\
\hline
\end{tabular}

nated drugs represented products that were listed on standard list of key drugs.

The median time from date of receiving to date of expiration for drug items was 485 days for NGO A and 420 days for NGO B. Figures $\mathbf{1}$ and $\mathbf{2}$ illustrate the time trends in median time of remaining time to expiration at the date of receiving by two NGOs. A time-series simple linear modeling of the data suggest downward trend overtime, indicating a decline of approximately $18( \pm 16)$ days every two months for NGO A and $13( \pm 7)$ days every two months for NGO B.

All six sampled NGOs were reported that, they did not have formal written policies. Most of NGOs in Sudan reported informal statements pertaining to the WHOModel List of Essential Drug (WHO-ML) and National Essential Drug List. NGOs in Sudan did not accept returned and dispensed drugs, but may accept drug samples 


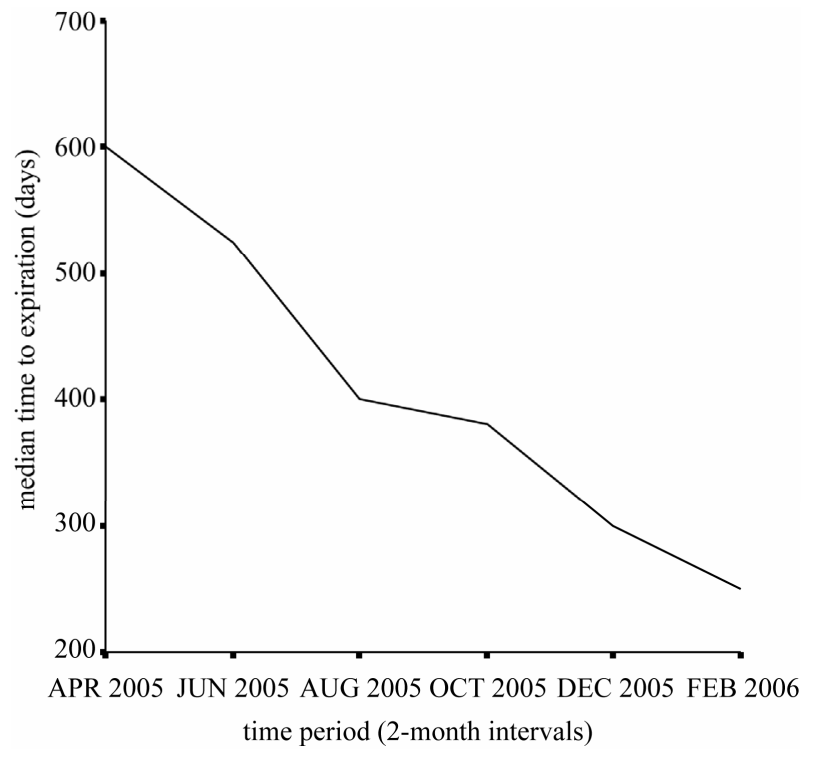

Figure 1. Median time between received by NGO A and expiration.

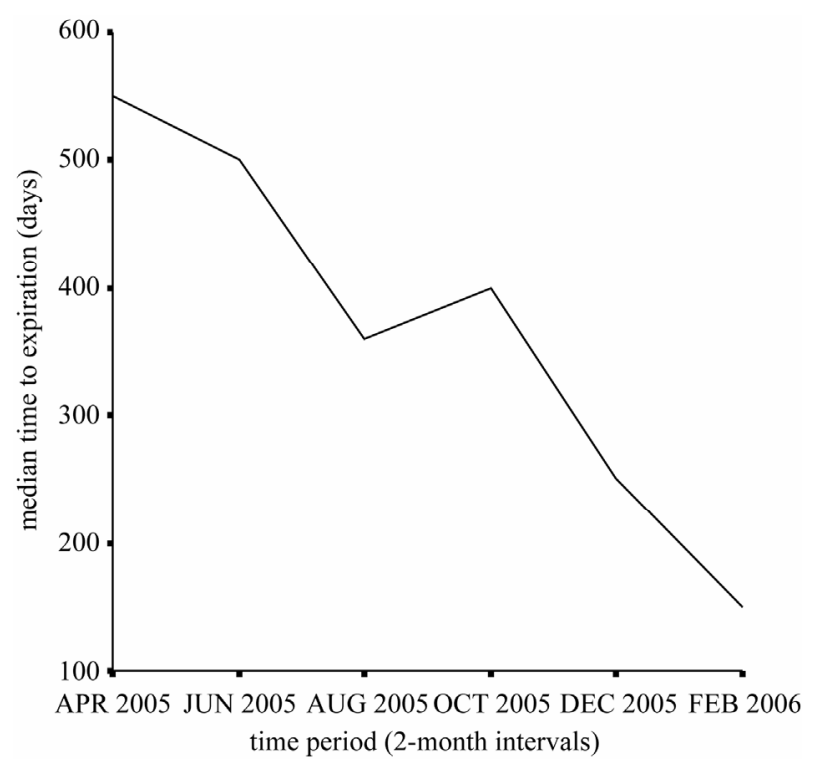

Figure 2. Median time between received by NGO B and expiration.

with some kind of safety and quality guarantee. The study concerning packaging, labeling and shipping costs showed that NGOs' statements were, on average, good and fair. Most of them specify in their policy statements that the labeling must be provided in language appropriate for the end-user. All of them reported that they take responsibility for paying all donation expenses, or they first attempt to secure funding from donating companies.

The field survey study revealed that, Sudan currently regulates the purchase, manufacture, importation, and use of pharmaceuticals based on National Drug Policy (NDP), and National List of Essential Drug (NEDL). Sudan did not develop its own Guidelines for Drug Donations. Instead, the National Drug Policy refers to the WHO Guidelines for Drug Donations. The majority of the policy regulations are enforced by General Directorate of Pharmacy (GDOP), either through its inspection unit or in its quality control laboratory. The Federal Ministry of Health through the Department of International Health and General Directorate of Pharmacy (GDOP) provide screening, coordination, and regulatory framework for all donated drugs enter the country. The FMOH, and NGOs Health Facilities officers feel that the advantages of the current donation processes outweigh its drawback.

\section{Discussion}

The study revealed that a large proportion of donated drugs met certain indicators of relevance. On the other hand, the study identified minor problems with relevance, as expressed by the presence of drugs not listed on the NEDL, drugs not labeled in INN, and drugs not labeled in locally understood language. Comparing the results of the current study with those of the Indonesian survey on drug donations that was carried out by Anonymous (2004) [7]; the results revealed that, $60 \%$ of donated drugs were not on the Indonesian List of Essential Drugs, and 70\% of donated drugs were labeled in language that is not locally understood by health professionals in Indonesia.

WHO Guideline No. 6 stated that: after arrival in recipient country all donated drugs should have a remaining shelf life of at least one year [3]. The current study was revealed partial adherence to these guidelines; $44.8 \%$ and $47.5 \%$ of the donated drugs for NGO A and NGO B respectively arrived with less than one year remained shelf life. The Srilanka study revealed a worse situation that $50.5 \%$ of total donations even had no labeled expiry date [8]. A survey by the WHO of 108 drug donations lists submitted to Albanian Ministry of Health during 1999 revealed that, about $41 \%$ of the drugs had a remaining shelf life of less than one year [9]. A significant proportion from $40.5 \%$ to $48.7 \%$ of donated drugs to two NGOs data bases, represented products that were listed on standard list of key drugs. This result suggested that minority of the donated drug products were not relevant to the local settings. The long field experiences of the NGOs in the country play important role in this product selection. The median time from date of receiving to date of expiration for drug items was 485 days for NGO A and 420 days for NGO B. The time trends in median time of remaining time to expiration at the date of receiving by two NGOs showed downward trend overtime. This pattern if persist will decline this measure of quality (time to expiration). Although the reasons for this secular trend may seems to be that: pharmaceutical companies give overstock drugs, collection of drugs from 
group and individuals in developed countries, and adhering by pharmaceutical companies and NGOs to Industry Principles Guidelines (1998) [10]. That permitted the offering of drugs with six months remaining shelf-life as donations, and minimal effort from local authorities regarding remaining shelf life of donated drugs. Bonati, 1996 reported that in Bosnia and Herzegovina between 1992-1996, a team of European Union hazardous waste experts found slimming agents from Britain, toxic chemicals with an expiry date of 1961, from the former East Germany, and nasal decongestant from the US with expiry date of 1990, which had been covered by a sticker saying 1993 [11].

All NGOs in the study sample reported that they did not have formal policies (written policies). A comparison of findings of Arnold and Reich (1990) [12] suggests that no progress has been made, at that time; five of eight NGOs contacted reported having formal policies or written policies, while in contrast, none of the six NGOs contacted for the current study provided some form of written policy statements.

Overall, the study identified important benefits of drug donations, as well as areas for improvement, from the perspective of the recipient. If these six categories are used to define the "relevance of donated drugs to local disease patterns, and national pharmaceutical priorities, then these results suggested that the majority of drugs selected for donations in this sample were relevant”.

The field survey revealed that Sudan currently regulates the purchase, manufacture, importation, and use of pharmaceuticals with its National Drug Policy (NDP), and National List of Essential Drug (NEDL). Sudan did not develop its own Guidelines for Drug Donations; instead, the National Drug Policy refers to the WHO Guidelines for Drug Donations. The majority of the policy regulations are enforced by General Directorate of Pharmacy (GDOP), either through its inspection unit or in its quality control laboratory. The Federal Ministry of Health through the Department of International Health and General Directorate of Pharmacy (GDOP) provided screening, coordination, and regulatory framework for all donated drugs enter the country. The FMOH, and NGOs Health Facilities' officers feel that the advantages of the current donation processes outweigh their drawbacks.

Finally the study recommended that, effort should be made to improve two-way communication and transparency among NGOs, recipient governments and recipient health facilities. FMOH should design a national policy for drug donations, in collaboration with WHO, and make this policy together with the NEDL available to NGOs.

The study forecasted that the problems of drug donations may be improving overtime. This could be achieved by informing donors about the adopted guidelines, with emphasis on development of performance standards on Good Donation Practices (GDP) for pharmaceuticals and to develop written policies for drug donations. The locally agreed performance standards and policy statements should be available to donors. The current study revealed that some inconformity to WHO Guideline No.6: which clearly stated that after arrival in recipient country all donated drugs should have a remaining shelf life of at least one year [3].

NGOs should be involved in dealing with expiration date and essential drug problems through workshops, seminars, donation meeting expressing needs, duration of shelf life, packaging and labeling, improvement of practical and ethical framework for donations, awareness on communication and establishment of donation standards.

Finally, the current study suggested that: introduction of National Essential Drug List a long with efforts from the International Health Department, and General Directorate of Pharmacy to review and approve the distribution of donations. Efforts should be carried out by the Office of Pharmacy Administration, Khartoum State to inspect NGOs health facilities and warehouses. NGOs should abide by the National Drug Policy (NDP), and to use their field experiences for drug selection and evaluation of overall donation process (some NGOs worked in Sudan for more than ten years operating on-going donation programs), all these contributed to better performance.

\section{Acknowledgements}

Authors would like to acknowledge the sincere cooperation that was offered by NGOs, General Directorate of Pharmacy Administration, Department of International Health and to the General Manager of Central Medical Supplies, Sudan.

\section{REFERENCES}

[1] H. V. Hogerzeil, M. R. Couper and R. Gray, "Guidelines for Drug Donations,” British Medical Journal (BMJ), Vol. 314, 1997, p. 737. doi: 10.1136/bmj.314.7082.737

[2] “Australian Guidelines for Drug Donations to Developing Countries,” Amendments Endorsed, November 2000

[3] “Guidelines for Drug Donations,” WHO/EDM/PAR/99.4 Revised, 1999.

[4] E. Hoen and C. Hodgkin, "Harmful Use of Donated Veterinary Drug,” Lancet, Vol. 2, 1993, pp. 308-209. doi: 10.1016/0140-6736(93)91856-H

[5] B. Snell, "Inappropriate Drug Donations: The Need for Reform,” Lancet, Vol. 758, 2001, p. 9281.

[6] M. R. Riech, "Pharmaceutical Donations: Players, Processes, and Products,” Assessment of US Pharmaceutical Donations, 1999.

[7] Pharmacien Sans Fronteire Comite International, “Acces- 
sibility for Everyone to Quality Pharmaceutical Care,” Press Release, 2004.

[8] Srilanka Ministry of Health, "Most Tsunami Drug Donations Were Useless and Dangerous (Report),” The Sunday times online, Vol. 41, No. 34, 2007, pp. 1391-0531.

[9] World Health Organization, "Think before You Send," WHO Regional Office for Europe, Press Release, Zagreb, 1999.

[10] WHO/EDM/PAR, "Guidelines for Safe Disposal of Unwanted Pharmaceuticals in and after Emergencies," Ge- neva: World Health Organization, WHO/EDM/PAR/98.2, 1998.

[11] M. Bonati, "Drug Donations to Bosnia-Herzegovina Are Often Inappropriate,” British Medical Journal (BMJ), Vol. 313, 1996, pp. 87-90. doi:10.1136/bmj.313.7063.1011b

[12] J. A. Patricia and M. R. Riech, "PVO Pharmaceutical Donations: Making the Incentive Fit the Need," Journal of Research in Pharmaceutical Economics, Vol. 804, 1990, pp. 40-60. 